\title{
Isolation and Characterization of cDNA Clones for Epidermis-Specific and Muscle-Specific Genes in Ciona savignyi Embryos
}

\author{
Shota Chiba ${ }^{1,2 *}$, Yutaka Satou ${ }^{1}$, Takahito Nishikata $^{2}$ \\ and Noriyuki Satoh ${ }^{1}$ \\ ${ }^{1}$ Department of Zoology, Graduate School of Science, Kyoto University, \\ Sakyo-ku, Kyoto 606-8502, Japan \\ ${ }^{2}$ Department of Biology, Konan University, Okamoto, Higashi-Nada-ku, \\ Kobe 658, Japan
}

\begin{abstract}
Ascidian eggs and embryos have provided an appropriate experimental system to explore the cellular and molecular mechanisms involved in the embryonic cell specification and pattern formation of the embryo. In Japan, most of the studies of ascidian embryology have been carried out with the large eggs of Halocynthia roretzi. However, for future studies, Ciona species may provide a better experimental system, in particular with respect to the incorporation of genetic approaches. In order to establish Ciona as an experimental system, molecular markers with which to examine cellular differentiation are required. In the present study, we isolated and characterized cDNA clones for two epidermis-specific genes (CsEpi-1 and CsEpi-2) and for two muscle-specific genes (CsMA-1 and CsMu-1). CsEpi-1 encodes a polypeptide with three trefoil domains, while CSMA-1 encodes a muscle-type actin from C. savignyi. Although CsEpi-2 and CsMu-1 transcripts seem to have a poly $(A)$ tail at the $3^{\prime}$ end, we could not find a distinct open reading frame in the sequences. Probes for CsEpi-1, CsMA-1 and CsMu-1 cross-reacted with C. intestinalis embryos. These cDNAs are useful as molecular markers for the specification of epidermis and muscle of Ciona embryos.
\end{abstract}

\section{INTRODUCTION}

Ascidian eggs and embryos have provided an appropriate experimental system to explore the molecular nature of localized maternal factors and their roles in cell specification and pattern formation (for reviews see Satoh, 1994; Satoh et al., 1996). The fertilized egg develops quickly into a tadpole larva, which consists of a small number of tissues including the epidermis, central nervous system with two sensory organs, nerve cord, endoderm, mesenchyme, notochord and muscle. The lineage of these embryonic cells is completely described up to the gastrula stage (Conklin, 1905; Nishida, 1987).

Recent molecular embryological studies have isolated and characterized cDNA clones for genes that are expressed in a tissue-specific manner, genes encoding transcriptional factors, and genes encoding signal molecules (reviewed by Chiba and Nishikata, 1998). In addition, recent studies have succeeded in the characterization of maternal genes with localized mRNAs, including posterior end mark (pem; Yoshida et al., 1996), pem-2, pem-4, pem-5, and pem-6 (Satou and Satoh,

\footnotetext{
* Corresponding author: Tel. +81-75-753-4095; FAX. +81-75-705-1113.
}

1997), and HrWnt-5 (Sasakura et al., 1998). One of the difficulties in ascidian molecular embryology is the need for techniques that deduce the function of these developmentally important genes. In some of the genes, the overexpression of the proteins produced by a microinjection of synthetic mRNA (Yoshida et al., 1996; Yasuo and Satoh, 1998) and the inhibition of the mRNA function by treatment with antisense oligonucleotides (Swalla and Jeffery, 1996; Olsen and Jeffery, 1997) resulted in distinct effects, providing cues to infer the gene functions. However, these techniques are not always successful.

We have speculated that genetic approaches such as those used for Drosophila, C. elegans and zebrafish could be applied to ascidians to identify genes with developmentally important functions. In Japan, most of the studies of ascidian embryology have been carried out with the large and transparent eggs of Halocynthia roretzi. However, H. roretzi may not be an appropriate system for future studies with genetic approaches. The spawning season is limited to winter, and the generation time may be more than two years. We propose Ciona eggs and embryos as an experimental system for further studies, because their spawning season is basically all year-round, and their generation time appears to be about 3 months (Kano and Amemiya, personal communication). 
Ciona savignyi or $C$. intestinalis could thus be useful as an experimental system for future studies, including those using genetic approaches.

In the present study, we therefore attempted to isolate cDNA clones for genes that are useful as molecular markers for the specification of embryonic cells. With such an aim, we used the subtractive hybridization of mRNAs of tailbud embryos with those of fertilized eggs. Taking advantage of the well-known lineage and segregation pattern of developmental fates as well as the in situ hybridization of whole-mount specimens, we were able to isolate cDNA clones for two epidermis-specific and two muscle-specific genes.

\section{MATERIALS AND METHODS}

\section{Ascidian eggs and embryos}

Ciona savignyi and $C$. intestinalis adults were collected near the Otsuchi Marine Research Center, Ocean Research Institute of the University of Tokyo, Iwate, Japan, and maintained under constant light to induce oocyte maturation. Eggs and sperm were obtained surgically from the gonoduct. After insemination, eggs were reared at about $18^{\circ} \mathrm{C}$ in Millipore-filtered seawater (MFSW) containing $50 \mu \mathrm{g} /$ $\mathrm{ml}$ streptomycin sulfate.

\section{RNA isolation and cDNA library construction}

Total RNA was isolated from fertilized eggs or tailbud embryos by the acid guanidinium thiocyanate-phenol-chloroform method (Chomczynski and Sacchi, 1987). Poly(A) ${ }^{+}$RNA was purified using Oligotex beads (Roche Japan, Tokyo). cDNA libraries of fertilized eggs (FE-library) and tailbud embryos (TB-library) were constructed in UniZAP XR using a ZAP-cDNA synthesis kit (Stratagene, La Jolla, CA, USA). A tailbud-mRNA concentrated subtractive library was constructed from TB- and FE-libraries as described by Satou and Satoh (1997).

\section{Screening of the subtracted cDNA library and sequencing}

From the library, clones were randomly picked up and partially sequenced from poly $(A)$ tail to avoid analyzing the same clones any further. After partial sequencing, each clone was examined for the localization of corresponding mRNA by whole-mount in situ hybridization using digoxigenin-labeled antisense RNA probes. Gastrulae and tailbud embryos were used as specimens for the in situ hybridization screening. cDNA clones exhibiting the localization of corresponding mRNAs were selected for further analyses.

Nucleotide sequences were determined for both strands with a dye primer cycle sequencing FS ready reaction kit and ABI PRISM 377 DNA sequencer (Perkin Elmer, Norwalk, CT, USA).

\section{Northern analysis}

Poly $(A)^{+}$RNA was isolated as described above and fractionated by agarose gel electrophoresis, and transferred to a Hybond- $\mathrm{N}(+)$ membrane (Amersham, Buckinghamshire, UK). Blots were hybridized with ${ }^{32} \mathrm{P}$-random-labeled DNA probes in 6 X SSPE, $0.5 \%$ SDS, 5 $X$ Denhardt's solution, $100 \mu \mathrm{g} / \mathrm{ml}$ salmon sperm DNA, and $50 \%$ formamide. The filter was washed twice in $2 \times$ SSC/0.1\% SDS, and twice in $0.2 \times \mathrm{SSC} / 0.1 \% \mathrm{SDS}$ at $65^{\circ} \mathrm{C}$, and exposed to $\mathrm{X}$-ray film.

\section{Whole-mount in situ hybridization}

RNA probes were prepared with a DIG RNA labeling kit (Boehringer Mannheim, Heidelberg, Germany). Whole-mount in situ hybridization was performed as described previously (Satou et al., 1995). The control specimens hybridized with sense probes did not show signals above the background.

\section{RESULTS AND DISCUSSION}

\section{Isolation of cDNA clones for tissue-specific genes in $C$. savignyi embryos}

In order to obtain cDNA clones for genes that are expressed in a tissue-specific manner, we constructed a cDNA library of tailbud-embryo mRNAs subtracted with the fertilizedegg mRNAs of $C$. savignyi. The library was estimated to contain about 90,000 clones. From the library, clones were randomly selected and their nucleotide sequences were determined from the 3' end to prevent the further analysis of the same clones. Each clone was then examined for the localization of corresponding mRNA by whole-mount in situ hybridization. Gastrulae and tailbud embryos were subjected to in situ hybridization to determine the specific expression of the genes. We have examined 100 clones to date and were able to find cDNA clones for two epidermis-specific genes (CsEpi1 and CsEpi-2) and two muscle-specific genes (CsMA-1 and CsMu-1) of $C$. savignyi embryos, which are described below.

\section{Expression of the CsEpi-1 gene}

Sequence analysis. The nucleotide and predicted amino acid sequences of a cDNA clone for CsEpi-1 are shown in Fig. 1. The insert of the clone consisted of 2,653 nucleotides. The clone contained a single open reading frame (ORF) that predicted 741 amino acids. The calculated molecular mass (Mr) of the CsEpi-1-encoded protein (CsEpi-1) was 81.9 k. A Northern blot showing a transcript of about $3.0 \mathrm{~kb}$ (Fig. 3) suggested that the clone contains all the coding sequences and is close to full-length.

As shown in Fig. 1, the sequence motif search using the Block Searcher (http://www.blocks.fhcrc.org/blockssearch.html) suggested that CsEpi-1 contains three P-type trefoil domains in the C-terminal half. Thim (1989) pointed out that four peptides present in completely different biological sources have been shown to exhibit a large degree of structural similarity. The peptides include the breast cancer-associated pS2 peptide isolated from human gastric juice and culture media of the human breast cancer cell line MCF-7 (Jakowlew et al., 1984), the pancreatic spasmolytic polypeptide (PSP) isolated from porcine pancreas (Tomasetto et al., 1990), and the peptide predicted from a cDNA isolated from the skin of Xenopus laevis (Hoffmann, 1988). The domain contain 6 cysteine residues in nearly the same positions, and these 6 residues are linked by 3 disulphide bonds to form a characteristic "trefoil" disulphide loop structure, as shown in Fig. 2b. Several studies have shown the presence of the trefoil domain in peptides abundantly produced at the mucousal surface of various animals (e.g., Hauser et al., 1992; Podolsky et al., 1993). Figure 2a shows a comparison of the amino acid sequence of the trefoil domain of CsEpi-1 with those of human intestinal trefoil hITF (Podolsky et al., 1993), human pS2 (Jakowlew et al., 1984), PSP (Tomasetto et al., 1990), the Xenopus laevis skin protein FIM-A.1 (Hoffmann, 1988), and another Xenopus laevis skin protein XP2 (Hauser et al., 1992). These domains shared the consensus sequences (Hoffmann 


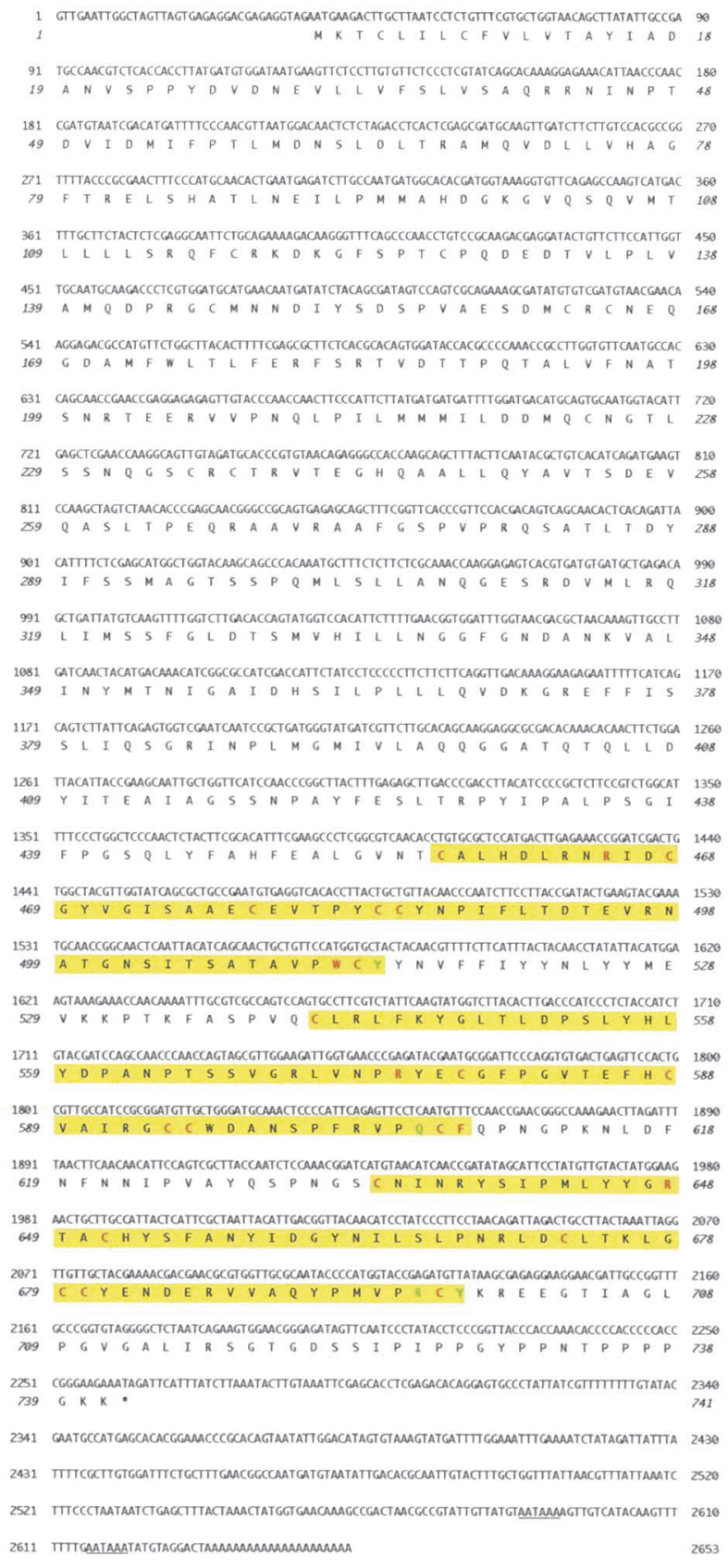

and Hauser, 1993).

The present report may be the first report of a trefoil family protein from invertebrates, although another trefoil family protein has been isolated from a colonial ascidian (Dr. Kazuo Kawamura, personal communication). During ascidian embryogenesis, epidermal cells produce larval and adult tunics. The tunic consists of mucus substances. CsEpi-1 may be a component of such mucus substances.

Spatial expression of CsEpi-1. The in situ hybridization of whole-mount specimens demonstrated that no signal was detected by the early gastrula stage (Fig. 4a) and that the first distinct signal was detected at the neurula stage (Fig. 4b). At this stage, the hybridization signal was evident in the nuclei of almost all of the epidermal cells. This signal was retained by the epidermal cells of the early tailbud embryos (Fig. 4c). A cross-section of hybridized embryos clearly showed that the CsEpi-1 expression was restricted to epidermal cells (Fig. 4d).

Cross-reactivity with Ciona intestinalis embryos. When we examined whether the CsEpi-1 antisense probe cross-reacts with $C$. intestinalis embryos, it became clear that the probe cross-reacted with $C$. intestinalis embryos (Fig. 5a). Thus, this gene is a useful molecular marker for epidermal cell differentiation in embryos of both Ciona species.

\section{Expression of the CsEpi-2 gene}

Sequence analysis. Nucleotide sequence of a cDNA clone for CsEpi-2 is shown in Fig. 6. The insert of the clone consisted of 1,618 nucleotides. There was a putative signal sequence for polyadenylation. In addition, the sequence in-

Fig. 1. Nucleotide and predicted amino acid sequences of a cDNA clone for CsEpi-1. The insert of the cDNA clone consists of 2,653 bp, with a single ORF that encodes a polypeptide of 741 amino acids. The asterisk indicates the termination codon. Two potential signal sequences for polyadenylation are underlined. The putative P-type trefoil domains are enclosed by yellow boxes. The amino acid residues that are conserved to form the P-type trefoil domain (see Fig. 2) are shown with red or green capitals. The accession number for the sequence of CsEpi-1 is AB008818 in the DDBJ, EMBL and GenBank nucleotide sequence databases.

$\mathbf{a}$

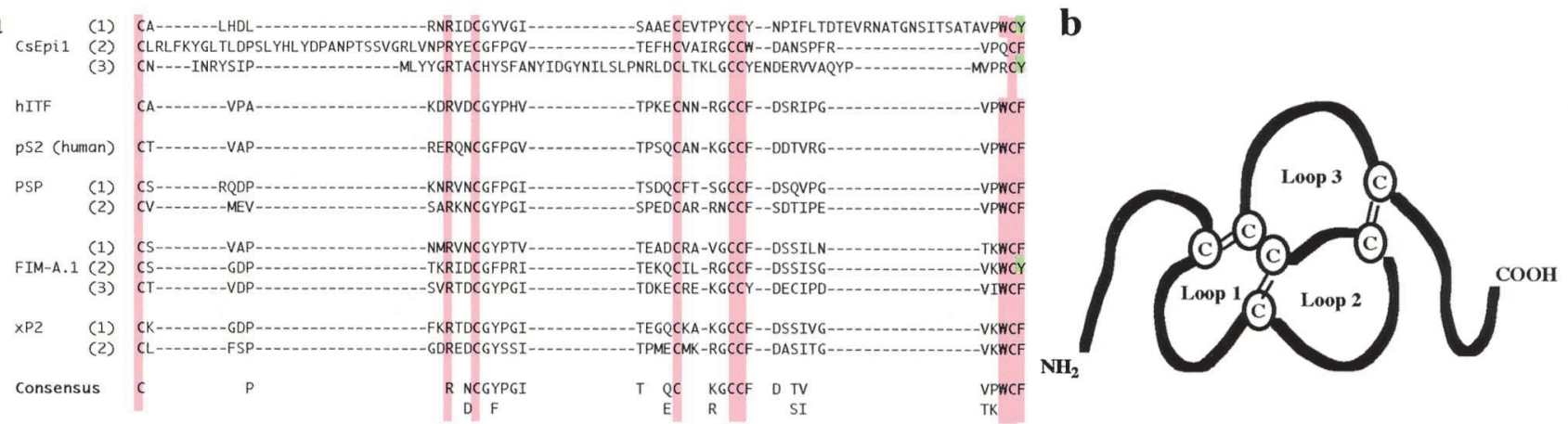

Fig. 2. (a) Comparison of amino acid sequences of the putative P-type trefoil domains of CsEpi-1 with those of other trefoil family members. Dashes indicate amino-acid residues without conservation. Sources: hITF (human, L08044; Podolsky et al., 1993), pS2 (human, X00474; Jakowlew et al., 1984), PSP (pig, X51696; Tomasetto et al., 1990), FIM-A.1 (Xenopus, M19971; Hoffmann, 1988), and xP2 (Xenopus, M90095; Hauser et al., 1992). The consensus was adopted from Hoffmann and Hauser (1993). (b) The predicted secondary structure of trefoil family proteins, adopted from Thim (1989). 
cluded 31 adenylyl residues at the 3' end, suggesting that the transcript has a poly(A) tail (Fig. 6). However, we could not detect any distinct ORF in the cDNA (Fig. 6). A Northern blot analysis, shown in Fig. 3, demonstrated that CsEpi-2 was not expressed in fertilized eggs but the transcript of about $1.7 \mathrm{~kb}$ was evident in the tailbud embryos. In addition, as described

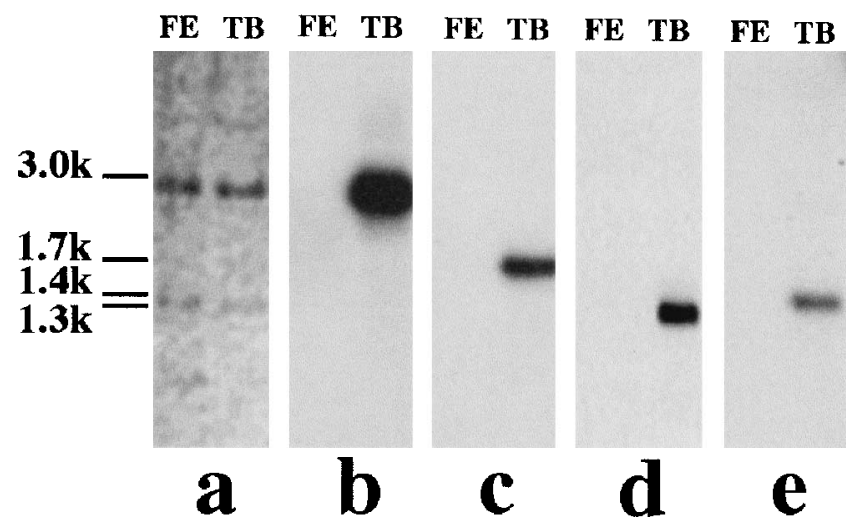

Fig. 3. Occurrence of transcripts of (b) CsEpi-1, (c) CsEpi-2, (d) CsMA-1 and (e) CsMu-1 in C. savignyi tailbud embryos. (a) Control gel. Northern blots of poly $(A)^{+}$RNA prepared from fertilized eggs (left lanes, FE) and tailbud embryos (right lanes, TB) were hybridized with the random-primed $\left.{ }^{32} \mathrm{P}\right]$-labeled DNA probes, and the membranes were washed under high-stringency conditions. Each lane was loaded with $1.5 \mu \mathrm{g}$ of poly $(\mathrm{A})^{+} \mathrm{RNA}$.

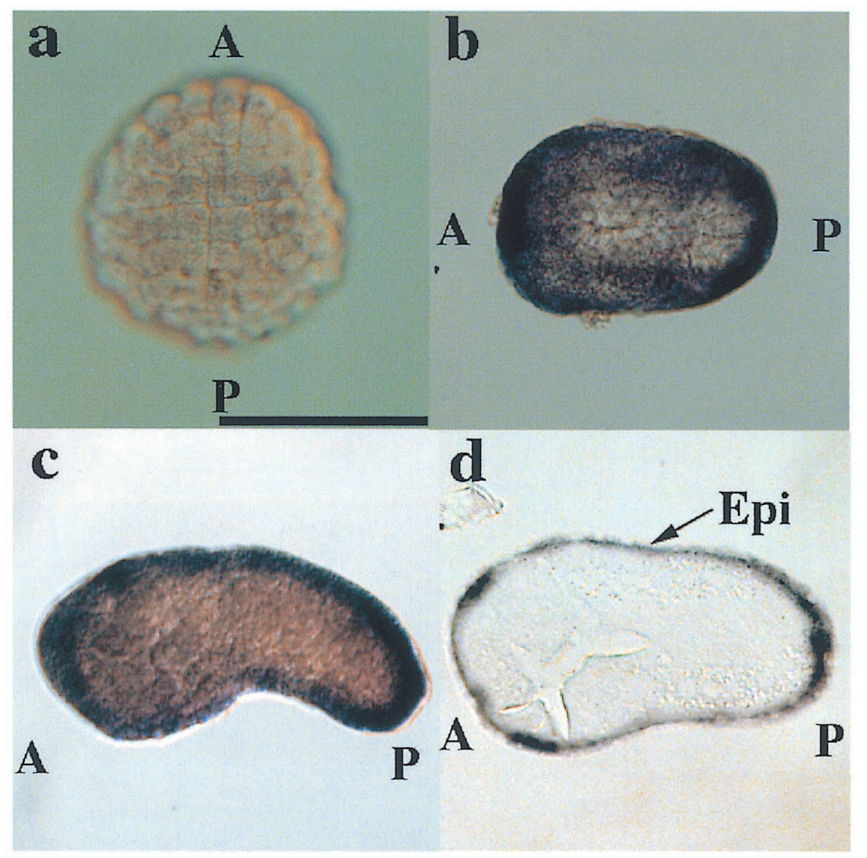

Fig. 4. Spatial expression of CsEpi-1, as revealed by whole-mount in situ hybridization. (a) An embryo at about the 110-cell stage viewed from the animal pole (future dorsal side of the embryo). No hybridization signal is detected at this stage. $\mathrm{A}$, anterior; $\mathrm{P}$, posterior. Scale bar represents $100 \mu \mathrm{m}$ for all panels. (b) A neurula, dorsal side view showing distinct signal in the epidermal cells. (c) An early tailbud embryo showing the signal in epidermal cells. (d) A sagittal section of the hybridized embryo showing that the signal is restricted to epidermis (Epi). below, an in situ hybridization showed that the CsEpi-2 transcript was evident in the nuclei of the 8-cell embryos. All of these data suggest that CsEpi-2 is expressed zygotically in C. savignyi embryos. We repeated the isolation and sequence determination of three independent clones corresponding to CsEpi-2, which showed sequence identity with a few differences.

As mentioned above, the CsEpi-2 transcript has no distinct ORF. We therefore examined possible secondary structures of CsEpi-2 transcript by calculation with the version 2.3 of Mfold (Zuker, 1989; Zuker and Jacobson, 1995). The predicted secondary structures of the CsEpi-2 transcript are shown in Fig. 7a.

Spatial expression of CsEpi-2. In most cases of zygotic expression of ascidian genes, the detection of mRNA by in situ hybridization of whole-mount specimens is more sensitive than that by Northern hybridization. This is because in situ hybridization can detect signals first in the nucleus of cer-
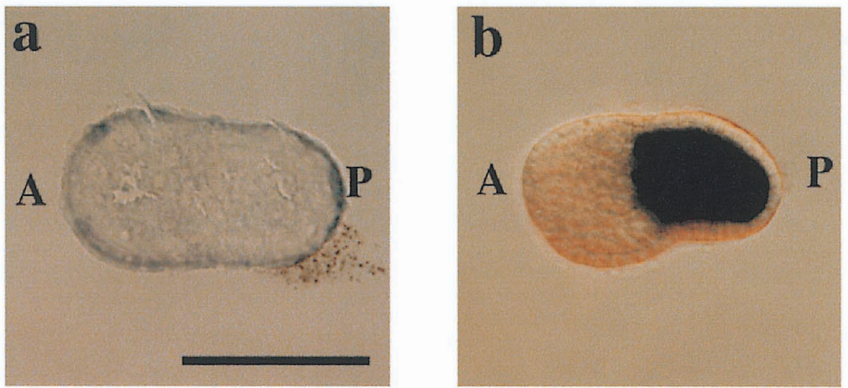

Fig. 5. Cross-reactivity of C. savignyi CsEpi-1 (a) and CsMA-1 (b) probes with $C$. intestinalis embryos, as revealed by whole-mount in situ hybridization. (a) The CsEpi-1 probe identified epidermal cells of C. intestinalis embryo (section), and (b) the CsMA-1 probe identified muscle cells of $C$. intestinalis embryo (whole).

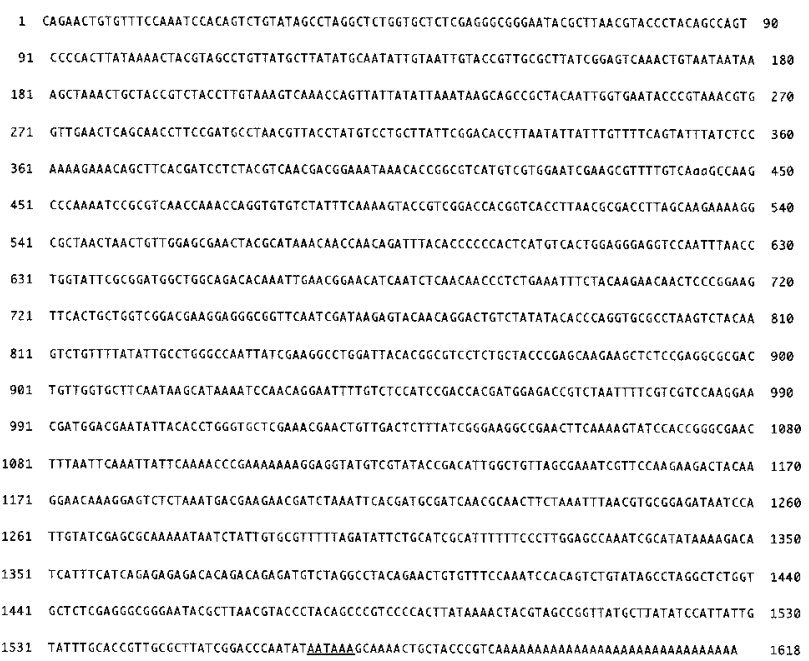

Fig. 6. Nucleotide sequence of a cDNA clone for CsEpi-2. The insert of the cDNA clone consists of $1,618 \mathrm{bp}$. The potential signal sequence for polyadenylation is underlined. The accession number for the sequence of CsEpi-2 is AB008820 in the DDBJ, EMBL and GenBank nucleotide sequence databases. 


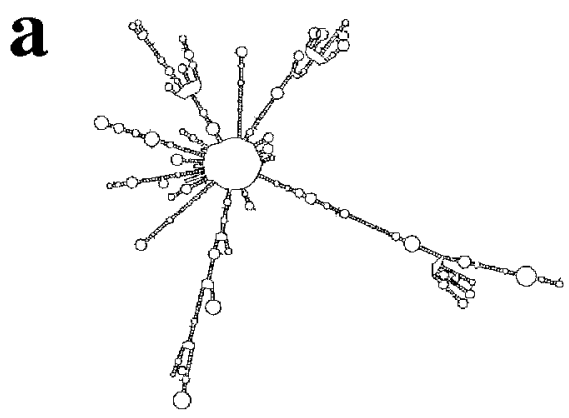

b

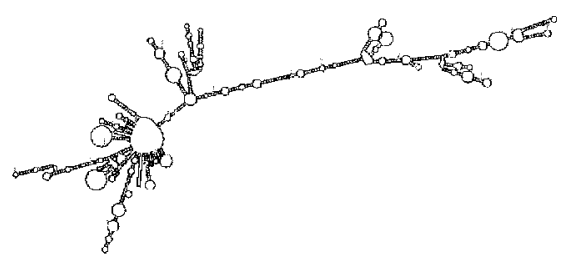

Fig. 7. Predicted secondary structures of (a) CsEpi-2 and (b) CsMu-1 transcripts.

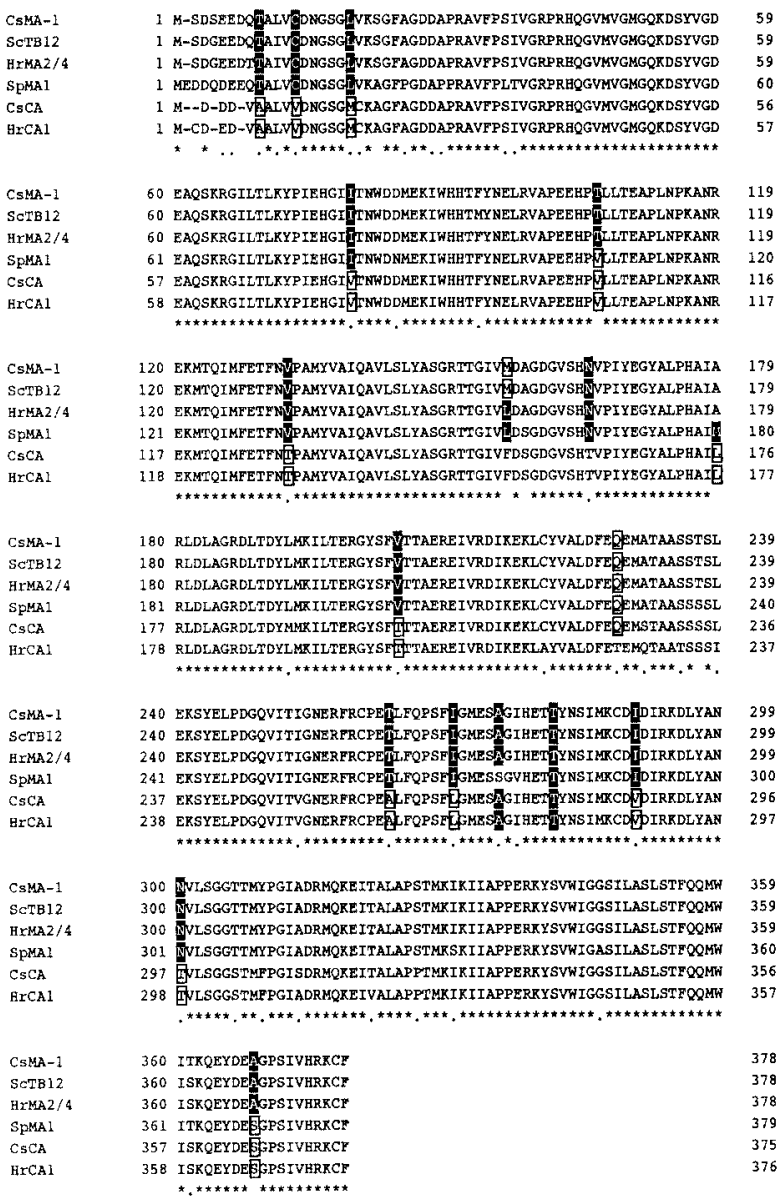

Fig. 9. The predicted amino acid sequences of a polypeptide encoded by a cDNA clone for CsMA-1, and comparison of the sequence with those of muscle-type (shown by dark boxes) and cytoplasmictype actins (shown by white boxes) of ascidians. The accession number for the sequence of CsMA-1 is AB008819 in the DDBJ, EMBL and GenBank nucleotide sequence databases. Sources: ScTB12 (Styela clava, muscle actin; Beach and Jeffery, 1992), HrMA2/4 (Halocynthia roretzi, muscle actin; Kusakabe et al., 1991), SpMA1 (Styela plicata, muscle actin; Kovilur et al., 1993), CsCA (Ciona savignyi, cytoplasmic actin; Y. Satou, unpublished data), and $\mathrm{HrCA} 1$ (H. roretzi, cytoplasmic actin; Araki et al., 1996).

tain cells which frequently develop in a lineage-specific and/ or region-specific manner (Yasuo and Satoh, 1993; Satou et al., 1995). This was the case for the CsEpi-2 gene.

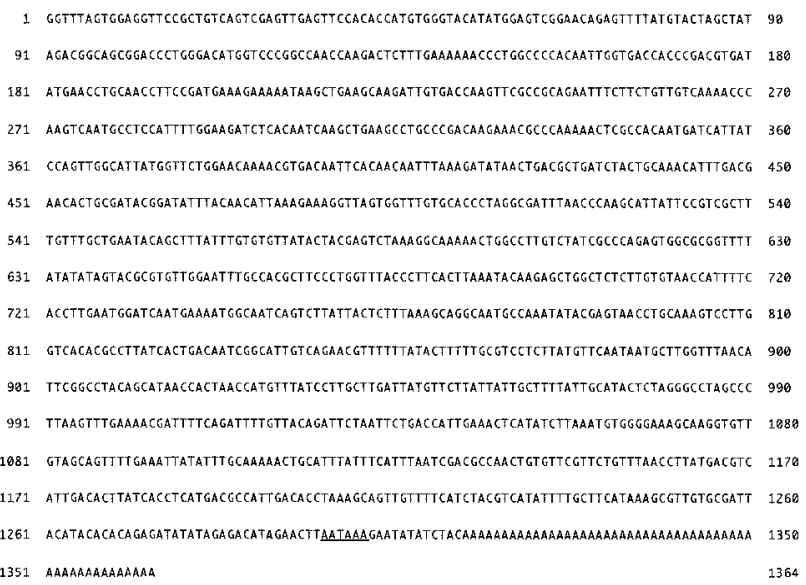

Fig. 11. Nucleotide sequence of a cDNA clone for CsMu-1. The cDNA clone consists of $1,364 \mathrm{bp}$. The potential signal sequence for polyadenylation is underlined. The accession number for the sequence of $\mathrm{CsMu}-1$ is AB008821 in the DDBJ, EMBL and GenBank nucleotide sequence databases.

The in situ hybridization demonstrated that the first distinct signal was detected as early as the 8-cell stage (Fig. 8). At this stage, the hybridization signal was evident in the nuclei of pairs of the a- and b-line primordial epidermal cells (Fig. 8a). During gastrulation and neurulation, the CsEpi-2 expression was retained only by epidermal cells (Fig. 8b). This signal was evident in the epidermal cells of the early tailbud embryos (Fig. 8c). The cross-section of hybridized embryos clearly showed that the CsEpi-1 expression was restricted to epidermal cells (Fig. 8d).

In order to deduce the gene function, we treated embryos with CsEpi-2 antisense oligos, but we did not obtain any meaningful results. The CsEpi-2 antisense probe did not cross-react with $C$. intestinalis embryos (data not shown).

The initiation of the appearance of CsEpi-2 was as early as the 8-cell stage. The first detection of a zygotic expression of ascidian genes was of the forkhead/HNF-3 gene which was reported to be at the 16-cell stage (Corbo et al., 1997; Olsen and Jeffery, 1997; Shimauchi et al., 1997). The CsEpi-2 gene may therefore represent the first zygotic expression of ascidians. 


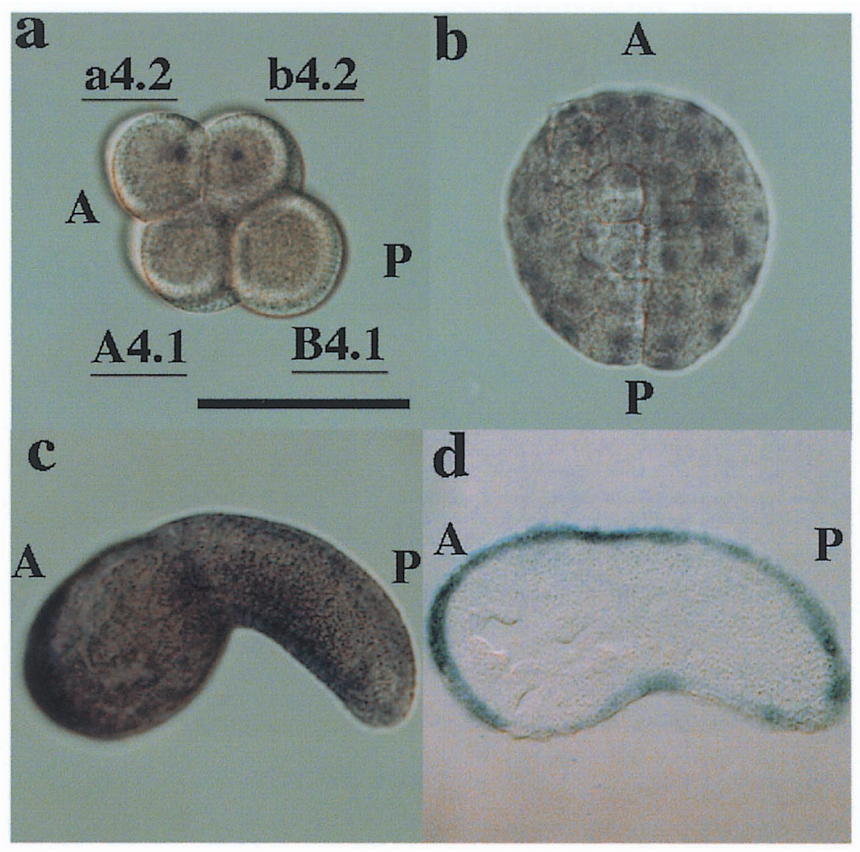

Fig. 8. Spatial distribution of CsEpi-2 transcript, as revealed by whole-mount in situ hybridization. (a) An 8-cell embryo, side view, showing that the hybridization signal is evident in the nuclei of a4.2 and b4.2 blastomeres, presumptive epidermal cells. A, anterior; P, posterior. Scale bar represents $100 \mu \mathrm{m}$ for all panels. (b) A gastrula viewed from the animal pole. Signal is found in the primordial epidermal cells. (c) An early tailbud embryo showing the signal in epidermal cells. (d) A sagittal section of the hybridized embryo showing that the signal is restricted to epidermis.

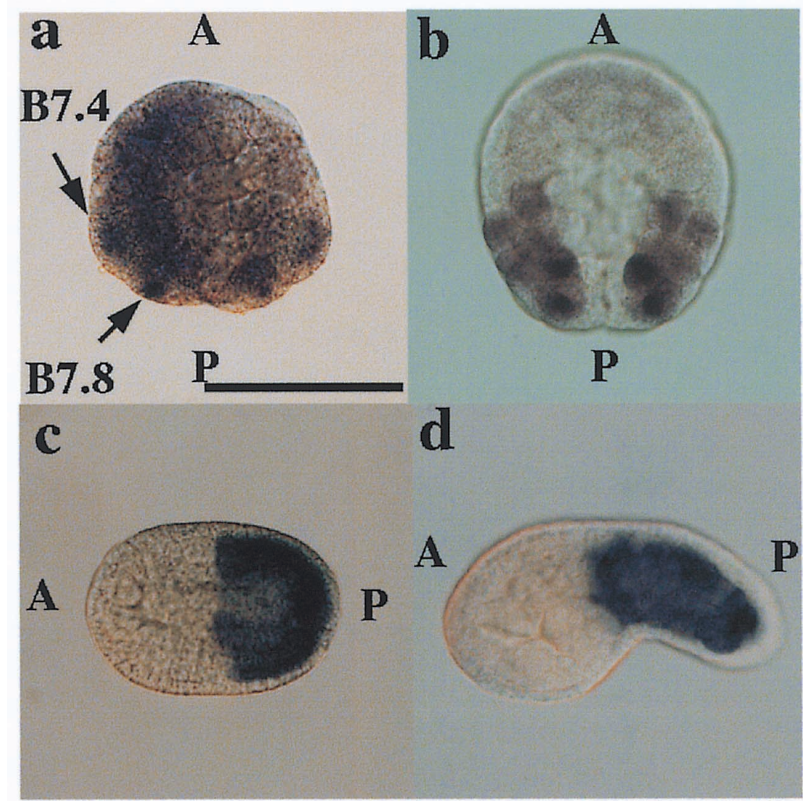

Fig. 10. Spatial expression of CsMA-1, as revealed by whole-mount in situ hybridization. (a) A 64-cell embryo viewed from the vegetal pole (future dorsal side of the embryo). Hybridization signal is evident in the nuclei of B7.4 and B7.8, the primordial B-line muscle cells. A, anterior; P, posterior. Scale bar represents $100 \mu \mathrm{m}$ for all panels. (b) A gastrula viewed from the vegetal pole showing the signal in the primordial muscle cells. (c) A neurula, dorsal side view, showing the signal in the primordial muscle cells. (d) An early tailbud embryo showing the signal in muscle cells of the tail region of the embryo.

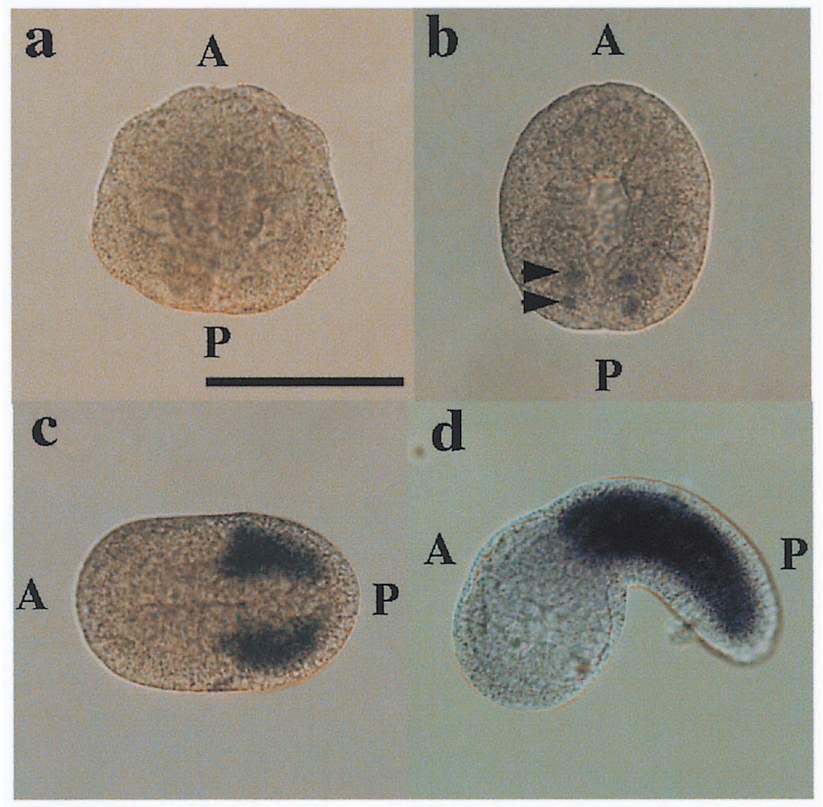

Fig. 12. Spatial distribution of $\mathrm{CsMu}-1$ transcript, as revealed by whole-mount in situ hybridization. (a) A 64-cell embryo viewed from the vegetal pole showing no hybridization signal. $A$, anterior; $P$, posterior. Scale bar represents $100 \mu \mathrm{m}$ for all panels. (b) A gastrula viewed from the vegetal pole showing the signal in two pairs of primordial muscle cells (arrowheads). (c) A late neurula, dorsal side view, showing the signal in the primordial muscle cells. (d) An early tailbud embryo showing the signal in muscle cells of the tail region of the embryo.

\section{Expression of a muscle-type actin gene CsMA-1}

Sequence analysis. The nucleotide sequence of the cDNA for CsMA-1 will appear under the accession number AB008817 in the DDBJ/EMBL/GenBank database. The insert of the clone consisted of 1,300 nucleotides including 21 adenylyl residues. The clone contained a single ORF that predicted 378 amino acids. Since (as shown below) the clone encodes a muscle actin, we designated this gene CSMA-1. The calculated molecular mass ( $\mathrm{Mr}$ ) of the CsMA-1-encoded protein (CsMA-1) was $42.1 \mathrm{k}$.

Most animals exhibit multiple actin isoforms which are encoded by a small gene family. In mammals, there are four muscle isoforms ( $\alpha$-skeletal, $\alpha$-cardiac, $\alpha$-vascular, and $\gamma$-enteric) and two nonmuscle isoforms ( $\beta$ - and $\gamma$-cytoplasmic) (Vandekerckhove and Weber, 1979) The mammalian $\alpha$-skeletal muscle actin is distinguishable from the $\beta$-cytoplasmic actin by about 20 diagnostic amino acid positions (Vandekerckhove and Weber, 1978, 1979). Figure 9 shows the comparison of the amino acid sequence of CsMA-1 with those of muscletype and cytoplasmic-type actin genes of ascidians. The comparison of the amino acid residues at the diagnostic positions indicated that the CsMA-1 is a muscle actin, while CsCA-1 is a cytoplasmic actin (Y. Satou, unpublished data).

Spatial expression of CSMA-1. The in situ hybridization demonstrated that the first distinct signal was detected at the 64-cell stage (Fig. 10a). The signals are evident in the nuclei of B7.4 and B7.8, the primordial B-line muscle cells. During gastrulation, signals became evident in B- (Fig. 10b), A-, and 
b-line presumptive muscle cells, and the neurulae showed signals in the primordial muscle cells (Fig. 10c). An early tailbud embryo showed distinct signal in muscle cells of the tail region of the embryo (Fig. 10d).

Cross-reactivity with Ciona intestinalis embryos. We confirmed that the CsMA-1 antisense probe cross-reacts with C. intestinalis embryos (Fig. 5b), and thus is useful as a molecular marker in that embryo.

The isolation of a $C$. savignyi muscle actin may provide material for future studies. We have already isolated a genomic clone of CsMA-1 and characterized the cis-regulatory elements required for the muscle-specific expression of CsMA-1.

\section{Expression of the CsMu-1 gene}

Sequence analysis. The nucleotide sequence of the cDNA clone of the CsMu-1 gene is shown in Fig. 11. The insert of the clone consisted of 1,364 nucleotides. There was a putative signal sequence for polyadenylation. In addition, the sequence included 51 adenylyl residues at the 3' end, suggesting that the transcript has a poly(A) tail (Fig. 11). However, as in the case of CsEpi-2, we did not detect any distinct ORF in the CsMu-1 cDNA (Fig. 11). The Northern blot analysis shown in Fig. 3 demonstrated that $\mathrm{CsMu}-1$ is not expressed in fertilized eggs, but the transcript of about $1.4 \mathrm{~kb}$ is evident in the tailbud embryos. In addition, the in situ hybridization showed that the CsMu-1 transcript is evident in the nuclei of the gastrula. Therefore, it is highly likely that $\mathrm{CsMu-1}$ is expressed zygotically in $C$. savignyi embryos. We examined four

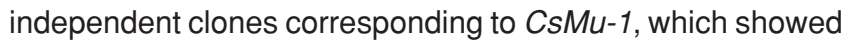
sequence identity with a few differences.

Similarity to the case of the CsEpi-2 transcript, we inferred possible secondary structures of the CsMu-1 transcript by calculation with the 2.3 version of Mfold. The predicted secondary structures of the CsMu-1 transcript are shown in Fig. 7b.

Spatial expression of CsMu-1. The in situ hybridization demonstrated that the first distinct signal was detected at the late gastrula stage (Fig. 12a, b). At this stage, the hybridization signal was evident in the nuclei of pairs of primordial muscle cells (Fig. 12b). During neurulation, the CsMu-1 expression expanded (Fig. 12c). An early tailbud embryo showed distinct signal in muscle cells of the tail region of the embryo (Fig. 12d). In order to deduce the gene function, we treated embryos with $\mathrm{CsMu-1}$ antisense oligos, but we did not obtain any meaningful results.

Cross-reactivity with Ciona intestinalis embryos. We examined whether the CsMu-1 antisense probe cross-reacts with $C$. intestinalis embryos. The probe identified muscle cells of C. intestinalis tailbud embryos (data not shown), and thus is useful as a molecular marker in that embryo.

\section{ACKNOWLEDGMENTS}

We thank Dr. Yasuaki Takagi and Mr. Koichi Morita and all staff of the Otsuchi Marine Research Center, Ocean Research Institute, University of Tokyo for their hospitality and their help in collecting ascidians. We also thank Kaz Makabe and Hiroki Takahashi for their critical reading of the manuscript.

YS was supported by a Predoctoral Fellowship from the Japan Society for the Promotion of Science (JSPS) for Japanese Junior Scientists with Research Grant \#8-6806. This research was supported by Research for the Future Program from the JSPS (96L00404) to TN and was also supported in part by a Grant-in-Aid for Specially Promoted Research (\#07102012) from the Ministry of Education, Science, Sports and Culture, Japan to NS.

\section{REFERENCES}

Araki I, Tagawa K, Kusakabe T, Satoh N (1996) Predominant expression of a cytoskeletal actin gene in mesenchyme cells during embryogenesis of the ascidian Halocynthia roretzi. Dev Growth Differ 38: 401-411

Beach RL, Jeffery WR (1992) Multiple actin genes encoding the same $\alpha$-muscle isoform are expressed during ascidian development. Dev Biol 151: 55-66

Chiba S, Nishikata T (1998) Genes of the ascidian: An annotated list as of July 1997. Zool Sci, in press

Chomczynski P, Sacchi N (1987) Single-step method of RNA isolation by acid guanidinium thiocyanate-phenol-chloroform extraction. Anal Biochem 162: 156-159

Conklin EG (1905) The organization and cell lineage of the ascidian egg. J Acad Nat Sci (Philadelphia) 13: 1-119

Corbo JC, Erives A, Gregorio AD, Chang A, Levine M (1997) Dorsoventral patterning of the vertebrate neural tube is conserved in a protochordate. Development 124: 2335-2344

Hauser F, Roeben C, Hoffmann W (1992) XP2, a new member of the P-domain peptide family of potential growth factors, is synthesized in Xenopus laevis skin. J Biol Chem 267: 14451-14455

Hoffmann W (1988) A new repetitive protein from Xenopus laevis skin highly homologous to pancreatic spasmolytic polypeptide. $J$ Biol Chem 263: 7686-7690

Hoffmann W, Hauser F (1993) The P-domain or trefoil motif : a role in renewal and pathology of mucous epithelia? Trends Biochem Sci 18: 239-243

Jakowlew SB, Breathnach R, Jeltsch JM, Masiakowski P, Chambon $P(1984)$ Sequence of the pS2 mRNA induced by estrogen in the human breast cancer cell line MCF-7. Nucleic Acids Res 12: 2861-2878

Kovilur S, Jacobson JW, Beach RL, Jeffery WR, Tomlinson CR (1993) Evolution of the chordate muscle actin gene. J Mol Evol 36: 361365

Kusakabe T, Suzuki J, Saiga H, Jeffery WR, Makabe KW, Satoh N (1991) Temporal and spatial expression of a muscle actin gene during embryogenesis of the ascidian Halocynthia roretzi. Dev Growth Differ 33: 227-234

Nishida H (1987) Cell lineage analysis in ascidian embryos by intracellular injection of a tracer enzyme. III. Up to the tissue restricted stage. Dev Biol 121: 526-541

Olsen CL, Jeffery WR (1997) A forkhead gene related to HNF-3 $\beta$ is required for gastrulation and axis formation in the ascidian embryo. Development 124: 3609-3619

Podolsky DK, Lynch-Devaney K, Stow JL, Oates P, Murgue B, DeBeaumont M, Sands BE, Mahida YR (1993) Identification of human intestinal trefoil factor. Goblet cell-specific expression of a peptide targeted for apical secretion. J Biol Chem 268: 66946702

Sasakura Y, Ogasawara M, Makabe KW (1998) HrWnt-5 : a maternally expressed ascidian Wnt gene with posterior localization in early embryos. Int J Dev Biol, in press

Satoh N (1994) Developmental Biology of Ascidians. Cambridge Univ Press, New York

Satoh N, Makabe KW, Katsuyama Y, Wada S, Saiga H (1996) The 
ascidian embryo: An experimental system for studying genetic circuitry for embryonic cell specification and morphogenesis. Dev Growth Differ 38: 325-340

Satou Y, Kusakabe T, Araki I, Satoh N (1995) Timing of initiation of muscle-specific gene expression in the ascidian embryo precedes that of developmental fate restriction in lineage cells. Dev Growth Differ 37: 319-327

Satou Y, Satoh N (1997) posterior end mark 2 (pem-2), pem-4, pem5 , and pem-6: Maternal genes with localized mRNA in the ascidian embryo. Dev Biol 192: 467-481

Shimauchi Y, Yasuo H, Satoh N (1997) Autonomy of ascidian fork head/HNF-3 gene expression. Mech Dev 69: 143-154

Sive HL, John TS (1988) A simple subtractive hybridization technique employing photoactivatable biotin and phenol extraction. Nucleic Acids Res 16: 10937

Swalla BJ, Jeffery WR (1996) PCNA mRNA has a 3' UTR antisense to yellow crescent RNA and is localized in ascidian eggs and embryos. Dev Biol 178: 23-34

Thim $L$ (1989) A new family of growth factor-like peptides. 'Trefoil' disulphide loop structures as a common feature in breast cancer associated peptide ( $\mathrm{pS} 2$ ), pancreatic spasmolytic polypeptide (PSP), and frog skin peptides (spasmolysins). FEBS Lett 250: $85-90$

Tomasetto C, Rio MC, Gautier C, Wolf C, Hareuveni M, Chambon P, Lathe R (1990) hSP, the domain-duplicated homolog of pS2 protein, is co-expressed with pS2 in stomach but not in breast carci- noma. EMBO J 9: 407-414

Vandekerckhove J, Weber K (1978) Mammalian cytoplasmic actins are the products of at least two genes and differ in primary structure in at least 25 identified positions from skeletal muscle actins. Proc Natl Acad Sci USA 75: 1106-1110

Vandekerckhove J, Weber K (1979) The complete amino acid sequence of actins from bovine aorta, bovine heart, bovine fast skeletal muscle, and rabbit slow skeletal muscle. A protein-chemical analysis of muscle actin differentiation. Differentiation 14:123133

Yasuo H, Satoh N (1993) Function of vertebrate T gene. Nature 364: 582-583

Yasuo H, Satoh N (1998) Conservation of the developmental role of Brachyury in notochord formation in a urochordate, the ascidian Halocynthia roretzi. Dev Biol, in press

Yoshida S, Marikawa Y, Satoh N (1996) posterior end mark; a novel maternal gene for a localized factors. Development 122: 20052012

Zuker M (1989) On finding all suboptimal foldings of an RNA molecule. Science 244: 48-52

Zuker M, Jacobson AB (1995) 'Well-determined' regions in RNA secondary structure prediction : analysis of small subunit ribosomal RNA. Nucleic Acids Res 23: 2791-2798

(Received January 13, 1998 / Accepted January 31, 1998) 